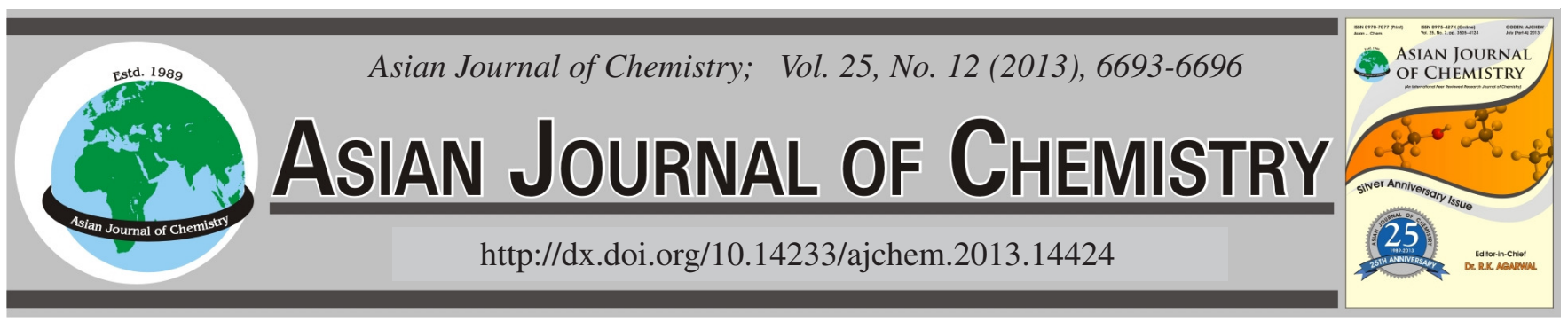

\title{
Determination of Fusarium Mycotoxin Beauvericin in Bombyx batryticatus by Liquid Chromatography-Tandem Mass Spectrometry (LC-MS/MS)
}

\author{
X. JiAng, Y.J. CHEN and L.G. SHI
}

College of Animal Sciences, Zhejiang University, Hangzhou, P.R. China

*Corresponding author: Tel/Fax: +86 571 88982723; E-mail: slgsilk@zju.edu.cn

(Received: 8 August 2012;

Accepted: 27 May 2013)

AJC-13551

\begin{abstract}
Fusarium Mycotoxin beauvericin has been proven to be a potent antibiotic compound useful for bio-control and a potential antifungal and anticancer agent for human. The aim of this study was to determine the level of Fusarium mycotoxin beauvericin in Bombyx batryticatus using LC-MS/MS and observe its content changes in production process within 12 days. The method yielded satisfactory precision, stability and repeatability (RSD: $0.63 \%, 0.96 \%, 1.16 \%$ ). Average recovery of three spiked levels was $100.3 \%$ with RSD $4.54 \%$. The level of beauvericin changes with a certain law in dead silkworm bodies. Fusarium mycotoxin beauvericin content was almost zero in the first four days, then extended increasing and reached its maximum in $10^{\text {th }}$ day, which was high up to $43.08 \mu \mathrm{g} / \mathrm{g}$. The trend of Fusarium mycotoxin beauvericin level declined from $10^{\text {th }}$ day. This is the first study to report the content of Fusarium mycotoxin beauvericin in $B$. batryticatus.
\end{abstract}

Key Words: Bombyx batryticatus, Fusarium Mycotoxin beauvericin, LC-MS/MS.

\section{INTRODUCTION}

Mycotoxins are secondary metabolites produced by different genera of filamentous fungi ${ }^{1}$, mostly Aspergillus, Fusarium and Penicillium ${ }^{2}$. Fusarium mycotoxin beauvericin is a small family of mycotoxins which is a cyclic hexadepsi-peptide consisting of three alternating $N$-methyl-L-amino acid and D- $\alpha$ hydroxyisovaleric acids (Fig. 1). Fusarium myco-toxin beauvericin was firstly isolated from a culture of the insect-pathogenic fungus Beauverina bassiana ${ }^{3}$, which was recognized as the causative agent for heavy losses of the sericulture. Beauvericin shows antibacterial ${ }^{4,5}$, antifungal, insecticidal activities ${ }^{6}$, even significant cytotoxic activity to various human cancer cell lines $^{7,8}$. Therefore, Fusarium mycotoxin beauvericin can be applied as a potential antibiotic and anticancer agent for human.

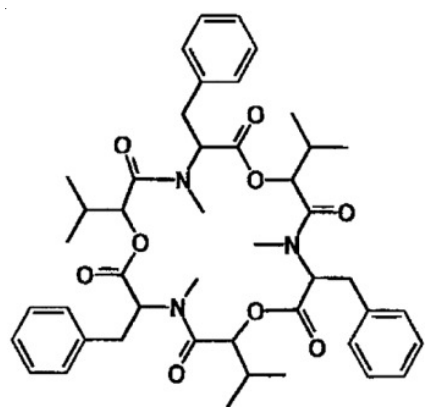

Fig. 1. Chemical structure of Fusarium mycotoxin beauvericin
B. batryticatus or Bombycis corpus, a batryticated and white-stiff silkworm, is a drug consisting of the dried larva of silkworm, Bombyx mori L, dead and stiffened due to the infection of Beauveria bassiana ${ }^{9}$. B. batryticatus has been always used in chinese traditional medicine to treat diseases such as palsy, headache, convulsion, stroke induced speech problem and tremor.

As $B$. bassiana can produce beauvericin, we speculate that $B$. batryticatus infected by the fungus contain a certain amount of beauvericin and its content changes in a regular way as time goes on. A number of methods have been developed to detect beauvericin in various matrices, however, is mainly focus on grain ${ }^{10,11}$ or food matrices ${ }^{12,13}$ and animal feed $^{2,14}$. The preparation methods are broadly similar including sample extraction of the analytes using different solvents and removal of impurities using different types of PSE columns. Up to date, we firstly determined beauvericin content in B. batryticatus in the present study. Besides, we presume that the content of beauvericin vary along with the growth of B. bassiana in silkworm and appear a definite rule. So we also investigated its content changes everyday within 12 days. There is almost no report on production of beauvericin besides Fusariun. redolens Dzf2 mycelial culture $^{15}$. Furthermore, this may supply another potential choice of large-scale production of beauvericin with lowcost and simple process. 


\section{EXPERIMENTAL}

Preparation of $\boldsymbol{B}$. batryticatus samples: B. batryticatus samples were made in May 2011. The silkworm larvae of strain (Qiufeng $\times$ Baiyu) were reared in mass on mulberry leaves up to the forth moult. Conidia of B. bassiana were collected from the confluent white fungal mat over the entire larval bodyandan inoculum was made up to contain approx. $1 \times 10^{7}$ conidia/ $\mathrm{mL}^{16}$. The inoculation was done by surface-application on to healthy larvae just after resumption of the fifth instar and the silkworms were reared continuously under more favourable circumstances $\left(25^{\circ} \mathrm{C}\right.$, humidity $\left.90 \%\right)$. The dead mummified larvae were collected and kept till they were covered with white conidiophores. Samples were collected at $24 \mathrm{~h}$ intervals. $B$. batryticatus were frozen-dried by Christpha 2-4 freeze drier (Bmh Instruments Co. Ltd.) prior to use.

Beauvericin standard was purchased from Sigma (St. Louis, MO, USA). Stock solution of $1 \mu \mathrm{g} / \mu \mathrm{L}$ of beauvericin were prepared in acetonitrile and stored at $-20^{\circ} \mathrm{C}$. Acetonitrile (Tedia, USA) was of LC quality. The water used was Milli-Q water purified with a Millipore Milli-Q Plus System. Sillica Solid Phase Extrsction columns (ProElut, 100 mg, $1 \mathrm{~mL}$ ) were purchased from Dikma (Beijing, China).

Sample preparation: B. batryticatus samples were ground with a laboratory mill. One gram sample was homogenized with $20 \mathrm{~mL}$ of $84 \%$ acetonitrile in water ${ }^{11,17}$ for $3 \mathrm{~min}$ and thereafter extracted for $0.5 \mathrm{~h}$ in an ultrasonic bath $(500 \mathrm{~W})$ for 2 times. The extracted samples were centrifuged at $8000 \mathrm{rpm}$ for $5 \mathrm{~min}$. Crude extracts were put together and homogenized. $1 \mathrm{~mL}$ crude extract was cleaned with SPE using a silica column ${ }^{5,12,18}$. Before loading the sample onto the column, the column was conditioned with $2 \mathrm{~mL}$ acetonitrile. A sample was passed through the conditioned column and the eluate was collected into a test tube. The column was further washed with $5 \mathrm{~mL}$ of acetonitrile, which was also collected. Eluates were pooled and concentrated under a stream of nitrogen, redissolved into $1 \mathrm{~mL} 50 \%$ acetonitrile in water and mixed thoroughly with a Vortex-Genie 2 test tube mixer. The dissolved sample was filtered through a $0.2 \mu \mathrm{m}$ syringe filter (Millpore). The samples were diluted to 100 times before injecting into a liquid chromatography-tandem mass spectrometer (LC-MS/MS).

A waters acquity ultra-performance liquid chromatography (UPLC) system coupled to a Quattro Ultima Pt Micromass Quattro Micro triple quadrupole mass spectrometer (Waters, Milford, MA, USA) equipped with an electrospray ionization (ESI) probe was used to analyze the samples. The analytical column was a $\mathrm{BEH} \mathrm{C}_{18}, 1.7 \mu \mathrm{m}, 2.1 \times 100 \mathrm{~mm}$ with inline filter, $0.22 \mu \mathrm{m}$ (Waters).

HPLC conditions: The column was kept at $40{ }^{\circ} \mathrm{C}$. The injection volume was $10 \mathrm{~mL}$. The mobile phase consisted of variable mixtures of mobile phase $\mathrm{A}$ (acetonitrile) and mobile phase $\mathrm{B}(10 \mathrm{mM}$ ammonium acetate) at a flow rate of $0.3 \mathrm{~mL} /$ min with a gradient elution program. The gradient elution started at $80 \%$ mobile phase B with a linear increase to $100 \%$ in $3 \mathrm{~min}$. This state kept for $0.5 \mathrm{~min}$ before mobile phase B was decreased to $80 \%$ during the next $0.1 \mathrm{~min}$ and then held for another $3 \mathrm{~min}$.
MS/MS detection: The mass spectrometer was operated in the positive electrospray ionization $\left(\mathrm{ESI}^{+}\right)$mode. The parameters of the MS were optimized using the standard solutions and the best response was recorded with the following parameters: cone voltage $45 \mathrm{~V}$, capillary voltage $3.80 \mathrm{kV}$, source temperature $150{ }^{\circ} \mathrm{C}$, desolvation temperature $350{ }^{\circ} \mathrm{C}$ and flow rate of desolvation $500 \mathrm{~L} / \mathrm{h}$. Nitrogen and argon were used as spray and collision gas, respectively. MRM-technique was used for identification and quantification, in which protonated molecule $[\mathrm{M}+\mathrm{H}]^{+}$of the analyte beauvericin $(\mathrm{m} / \mathrm{z}, 785)$ was fragmented in the collision cell to the product ions: $\mathrm{m} / \mathrm{z}$ 243.9 and 261.9. Product-ion $\mathrm{m} / z 243.9$ was used for the quantification of beauvericin. However, the quantification of beauvericin was achieved by comparing the peak areas of the samples with the corresponding calibration plot of the beauvericin standard (Fig. 2).
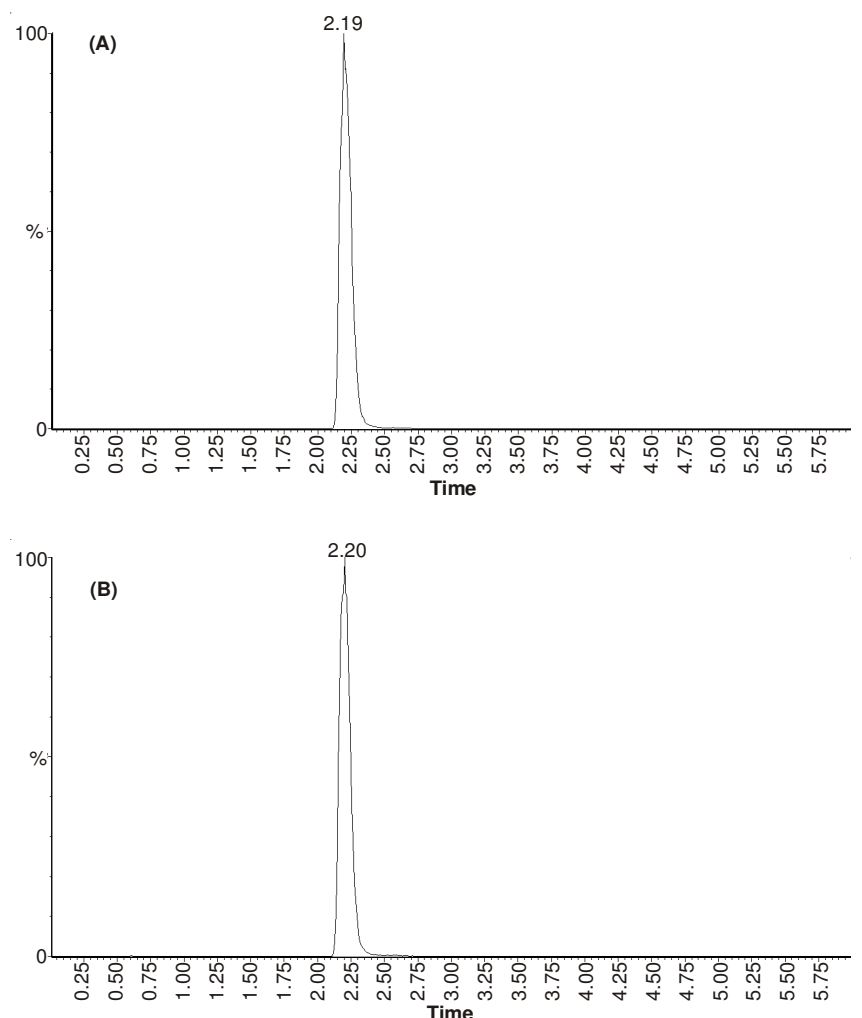

Fig. 2. HPLC chromatogram of beauvericin standard (A), B. batryticatus samples (B)

Method validation: Five validation parameters were determined to describe the performance of the method: Linearity, precision, stability, recovery and repeatability, limit of detection (LOD) and limit of quantification (LOQ). Signalto-noise $(\mathrm{S} / \mathrm{N})$ ratios of 3 or above were considered acceptable for LOD and $\mathrm{S} / \mathrm{N}$ of 10 or above for LOQ. Replicates $(\mathrm{n}=3)$ of spiked samples (the beauvericin content had already known in every matrix sample), at three concentration levels and calibration curve were analyzed.

\section{RESULTS AND DISCUSSION}

Purification and qualitative identification of beauvericin in $\boldsymbol{B}$. batryticatus: $84 \%$ acetonitrile in water, which is reported to receive the best recovery of beauvericin, was 
chosen to be the extraction solvent. Utilization of silica SPE overcomes shortcomings of loss-causing liquid-liquid extraction. Beauvericin does not have any chemical group that is easily accessible for derivatization, but it is able to form complexes with different metal ions such as $\mathrm{Na}^{+}, \mathrm{K}^{+}, \mathrm{Ca}^{+}$and $\mathrm{NH}_{4}{ }^{+}$, which could fragmented in various ways and lead to problems in quantification. By using ammonium acetate in the mobile phase, we could exclude all other complexes for beauvericin, except $\mathrm{M}+\mathrm{NH}_{4}$. In $\mathrm{MS}$ trace analysis, MRM mode was used, which means two specific ions produced by the analyte were detected.

Under the same HPLC-MS/MS condition, B. batryticatus was analyzed and the chromatogram results showed that one single peak was eluted with the same retention time of 2.20 min to standard solution. Besides, when spiked by beauvericin standard, chromatographic peak area increased linearly (Fig. 2 ). This shows that B. batryticatus contains beauvericin and beauvericin can be well separated by HPLC and analyzed qualitatively and quantitatively according to its retention time and peak area. Finally, the mean concentration of beauvericin was $21.64 \mu \mathrm{g} / \mathrm{g}$.

Method validation: Using the concentration of beauvericin standard solution as abscissa and the peak area as response, a better linear chart at the concentration of $5-1000 \mathrm{ng} / \mathrm{mL}$ was constructed: $\mathrm{y}=8270.03 \mathrm{x}+11519.5\left(\mathrm{r}^{2}=\right.$ 0.9973). Apparatus precision was evaluated by determination of $B$. batryticatus for five successive times and solution stability was by the analysis a solution of the sample every $2 \mathrm{~h}$. Spiked solutions were prepared in acetonitrile and appropriate amounts added to $B$. batryticatus samples. Repeatability of the method was evaluated by the analysis of three parallel B. batryticatus samples and recovery was by analyzing spiked samples at three concentration levels. The determined LOD and LOQ were calculated according to the guidelines of the German norm DIN 3264 which are derived from the y-intercept and its confidence interval after linear regression, which is $4 \mu \mathrm{g} / \mathrm{g}$ and 12 $\mu \mathrm{g} / \mathrm{g}$, respectively. In general, the method yielded satisfactory precision, stability and repeatability, whose RSDs were 0.63 , 0.96 and $1.16 \%$. Also a better mean recovery and precision for beauvericin was obtained from spiked B. batryticatus samples (Table-1).

\section{TABLE-1}

MEAN RECOVERIES OF BEA AT THREE DIFFERENT LEVELS

\begin{tabular}{lccc}
\hline Spiking level & $\begin{array}{c}\text { Level 1 } \\
(10 \mu \mathrm{g} / \mathrm{g})\end{array}$ & $\begin{array}{c}\text { Level 2 } \\
(30 \mu \mathrm{g} / \mathrm{g})\end{array}$ & $\begin{array}{c}\text { Level 3 } \\
(60 \mu \mathrm{g} / \mathrm{g})\end{array}$ \\
\hline Recovery (\%) & $104.3 \pm 3.15$ & $100.9 \pm 3.12$ & $95.7 \pm 2.70$ \\
Average recovery (\%) & $100.3 \pm 4.54$ & & \\
\hline
\end{tabular}

Changes of beauvericin content in silkworm bodies post-inoculation by $\boldsymbol{B}$. bassiana: The level of beauvericin in dead silkworm bodies changes with a certain law from 1 st to 12th day after inoculation presented in Fig. 3. In the previous 4 days beauvericin 's level was close to zero, then increase steadily until it reached the highest level in day 10 , at which point it dipped sharply in the next 2 days. The level of B. batryticatus sample was $21.64 \mu \mathrm{g} / \mathrm{g}$, which was between that of day 7 and day 8 . The highest content of day 10 reached $43.08 \mu \mathrm{g} / \mathrm{g}$ more than twice that of B. batryticatus sample. The next 2 days' content was almost as high as that of day 7 . Since the inoculated silkworms began to deteriorate form 9 th day, we speculated that migration downwards of beauvericin level in 11th day was because the life-cycle of the fungus was completed.

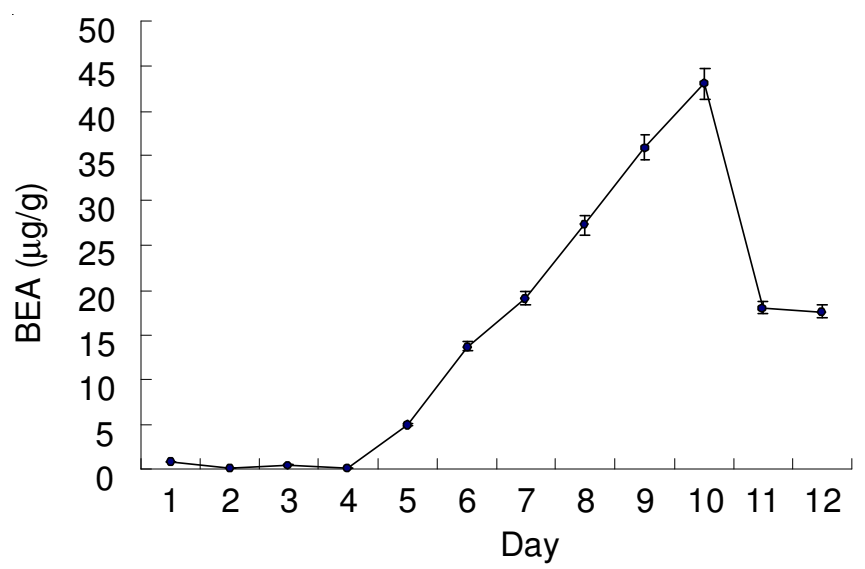

Fig. 3. BEA content in silkworm bodies inoculated with $B$. bassiana from fifth moult

\section{Conclusion}

Methods for the quantitative determination of mycotoxins in animal feed ${ }^{2}$, grains ${ }^{10}$ or eggs ${ }^{12}$ were already available. Based on those recommendations, this study has successfully shown the potential of LC-MS/MS method to rapidly determine beauvericin 's level in B. batryticatus. Compared to those published papers that detect beauvericin contamination in food matrices, the values of LOD and LOQ were higher. This is probably because of the sensitivity of LC-MS/MS to beauvericin in the fungus-insect complex. Also, the ingredients of the complex were too complicated so that matrix effect was a little stronger, which led to the higher LOD and LOQ.

The validation results showed that the sample preparation method developed for detecting beauvericin in food matrices was well applicable for the determination in B. batryticatus. The results showed $B$. batryticatus sample contained sufficient beauvericin which was as high as $21.64 \mu \mathrm{g} / \mathrm{g}$. Four days before the whole preparation of $B$. batryticatus beauvericin's level was almost close to zero and then beauvericin increased linearly with the passage of time before the 10th day. After that beauvericin 's level declined rapidly in 11th day and that of day 12 remained almost the same. So we figure that the change law of beauvericin 's level supports the growth rule of Beauverina bassiana within silkworm body as reported ${ }^{16}$. Kumar et al. ${ }^{16}$ confirmed that the life-cycle of Beauverina bassiana on silkworm was completed in 7-8 days. However beauvericin 's level reached the highest in day 10. Time difference between the paper and our practice may because of some reasons that lag the whole life-cycle including differences in infectious ability of the fungus conidia, varieties of silkworm, manual operation of inoculation and culture conditions.

Thus B. batryticatus samples contain sufficient beauvericin and we already know 10th day's level is the highest. This demonstrates the feasibility and promising potential of preparation of $B$. batryticatus for efficient and large-scale production of beauvericin and related bioactive compounds. 


\section{ACKNOWLEDGEMENTS}

This work was supported by the National Natural Science Foundation of China grant No. 31072090 and No. 31272375. We are grateful to Zengxuan Cai from Zhejiang Centers for Disease Control and Prevention for his technical support.

\section{REFERENCES}

1. C. Paciolla, M.P. Ippolito, A. Logrieco, N. Dipierro, G. Mule and S. Dipierro, Physiol. Mol. Plant. Pathol., 72, 3 (2008).

2. K.E. Lee, B.H. Kim and C. Lee, Anim. Feed Sci. Tech., 157, 190 (2012)

3. R.L. Hamill, C.E. Higgens, H.E. Boaz and M. Gorman, Tetrahedron Lett., 49, 4255 (1969).

4. L.A. Castlebury, J.B. Sutherland, L.A. Tanner, A.L. Henderson and C.E. Cerniglia, World J. Microbiol. Biotechnol., 15, 131 (1999).

5. G. Meca, I. Sospedra, J.M. Soriano, A. Ritieni, A. Moretti and J. Mañes, Toxicon., 56, 349 (2010).

6. J.F. Grove and M. Pople, Mycopathologia, 70, 103 (1980).

7. G.M. Jow, C.J. Chou, B.F. Chen and J.H. Tsai, Cancer Lett., 216, 165 (2004).
8. L. Calo, F. Fornelli, R. Ramires, S. Nenna, A. Tursi, M.F. Caiaffa and L. Macchia, Pharmacol. Res., 49, 73 (2004).

9. H.J. Kim, W.H. Lee, C.H. Yoon, J.C. Jeong, K.S. Nam, H.M. Kim, Y.K. Choo, M.C. Lee and C.H. Kim, Pharmacol. Res., 43, 11 (2001).

10. M.K. Kokkonen and M.N. Jestoi, Food Anal. Method., 2, 128 (2009).

11. S. Uhlig and L. Ivanova, J. Chromatogr. A, 1050, 173 (2004).

12. M. Jestoi, M. Rokka, E. Järvenpää and K. Peltonen, Food Chem., 115, 1120 (2009).

13. S. Monbaliu, C. Van Poucke, C. Van Peteghem, K. Van Poucke, K. Heungens and S. De Saeger, Rapid Commun. Mass Spectrom., 23, 3 (2009).

14. J.L. Sorensen, K.F. Nielsen, P.H. Rasmussen and U. Thrane, J. Agric. Food Chem., 56, 10439 (2008).

15. L.J. Xu, Y.S. Liu, L.-G. Zhou and J.-Y. Wu, Biotechnol. Bioproc. Eng., 15, 460 (2010)

16. V. Kumar, G.P. Singh, A.M. Babu, M.M. Ahsan and R.K. Datta, Ital. J. Zool., 66, 39 (1999).

17. M. Jestoi, M. Rokka, A. Rizzo, K. Peltonen and S. Aurasaari, J. Liq. Chromatogr. Rel. Technol., 28, 369 (2005).

18. M. Jestoi, M. Rokka and K. Peltonen, Mol. Nutr. Food Res., 51, 625 (2007). 